\title{
Le paysage montréalais dans La Brûlerie d'Émile Ollivier
}

\section{The Montreal Landscape in Émile Ollivier's La Brûlerie}

Petr Kyloušex [kylousek@phil.muni.cz]

Masarykova univerzita, République tchèque

\section{RÉSUMÉ}

Émile Ollivier est un des auteurs de la diaspora haïtienne et un de ceux qui appartiennent désormais à la littérature montréalaise et québécoise. Son œuvre illustre la complexité de la situation identitaire liée à l'émigration et à l'insertion (ou à l'absence d'insertion) dans les espaces civilisationnels d'accueil. La Brûlerie est un des romans qui accorde à l'espace et à l'identité spatialisée une attention particulière. Située au pied du Mont-Royal, avec vue sur le Lac Saint-Louis et le Saint-Laurent, tel un port au bord de la mer des Caraïbes, La Brûlerie est cet endroit hybride, proche de l'hétérotopie foucaultienne, où se côtoie le passé haïtien avec le présent multiple de la postmodernité et à partir duquel se déploie la sensibilité identitaire postmoderne - rhizomatique - de l'auteur.

\section{MoTS-CLÉS}

littérature québécoise; littérature migrante; diaspora haïtienne; identité spatiale

\begin{abstract}
Émile Ollivier, one of the authors of the Haitian diaspora, belongs to the Montreal and Quebec literature since the sixties. His work illustrates the complexity of the identity situation related to migration and integration (or to lack of insertion) in a host civilizational spaces. La Brûlerie is one of the novels that pay a special attention to space and spatial identity. Located at the foot of Mont-Royal, overlooking St. Louis Lake and St. Lawrence River, as if it were a port facing the Caribbean Sea, the café La Brûlerie is a hybrid place, an ideal Foucault's heterotopia. The past of Haiti crosses a miscellaneous, postmodern present from which the author's rhizomatic sensibility arises.
\end{abstract}

\section{KEYWORDS}

Quebec literature; migration literature; Haitian diaspora; spatial identity

REÇU 2015-08-31 ; ACCEPTE 2016-06-30 
Comment penser l'instabilité et le mouvement qui caractérisent le monde présent et qui sont probablement appelés à s'intensifier dans un proche avenir, sans pour autant perdre le rapport à la mémoire, à la continuité intellectuelle, au profit d'une exaltation du présent?

Émile Ollivier (1940-2002) est un des auteurs de la diaspora haïtienne et un de ceux qui appartiennent désormais à la littérature montréalaise et québécoise. Son œuvre illustre la complexité de la situation identitaire liée à l'émigration et à l'insertion (ou à l'absence d'insertion) dans les espaces civilisationnels d'accueil. La Brûlerie, son dernier roman, accorde à l'espace et à l'identité spatialisée une attention particulière. Située au pied du Mont-Royal, avec vue sur le Lac Saint-Louis et le Saint-Laurent, tel un port au bord de la mer des Caraïbes, le café La Brûlerie est cet endroit hybride, proche de l'hétérotopie foucaultienne (Foucault 1984), où se côtoie le passé haïtien avec le présent multiple de la postmodernité et à partir duquel se déploie la sensibilité identitaire postmoderne - rhizomatique - de l'auteur. Le néologisme de Thomas C. Spear qui désigne l'auteur comme un enracinerrant est sans doute pertinent (Spear 2002).

Résumons le questionnement existentiel et le doute noétique qui traversent le récit. L'intrigue se noue autour d'un groupe de six Haïtiens, amis de longue date, car anciens élèves de l'Institut français de Port-au-Prince (Ollivier 2004 : 71). Militants antiduvaliéristes, expatriés ou poussés à l'exil, ils habitent Montréal depuis des décennies. Leur "Ministère de la Parole " (Ollivier 2004 : 21 sqq.) siège au café La Brûlerie dans le quartier de la Côte-des-Neiges. Un beau jour, le personnage-narrateur Jonas Lazard est abordé par Cynthia, fille de Naomie et de Virgile qui, après la mort tragique de sa mère, fusillée par l'armée chinoise sur la place Tiananmen, à Pékin, en 1989, est à la recherche de son père qu'elle n’a pas connu. Or Virgile, foudroyé par la mort de Naomie, vient de se suicider au Royal Terrasse Hôtel, face au café. La quête de Cynthia déclenche le questionnement concernant cet ami de longue date et membre du Ministère de la Parole. En effet : «Qui était Virgile? Ce serait trop simple de répondre qu'il ressemblait trait pour trait à toute une génération écartelée entre poétique et politique, idéologie et foi, livre et fusil?» (Ollivier 2004 : 31) D’autant plus que

Depuis quelque temps, plus précisément depuis ce fameux jour de juin, date du massacre de la place Tiananmen, Virgile semblait avoir rompu tout contact avec lui-même et avec ses semblables. [...] Certains voulurent partir à sa recherche mais prirent conscience que, même si cela faisait des décennies qu'ils le rencontraient, leur amitié avec lui était sans intimité. Ils ne connaissaient ni son adresse ni son numéro de téléphone. (Ollivier 2004 : 60-61)

C'est autour de ce disparu, ce vide de la double mort et de la solitude, que se déroule l'investigation. ${ }^{2}$ Virgile pose question. Mais cette question vise chacun de ses amis, anciens militants de gauche : à chacun son exil, à chacun son errance, à chacun sa façon de vivre Montréal.

La remémoration, car tel est l'enjeu de la quête, est spatialisée, inscrite dans le paysage urbain de Montréal qui constitue le point nodal de la narration. En effet, par quels procédés l’espace

1 Ollivier, É. (2002). L'enracinement et le déplacement à l'épreuve de l'avenir. Études littéraires, 34, 3, 88.

2 Signalons à ce propos l'analogie avec certains romans de Patrick Modiano. Voir Voldřichová Beránková. (2004). 
réel se transforme-t-il en l'espace symbolique pour exprimer une expérience existentielle ? Quelle est la relation entre cette expérience de l'espace et la noésis et par-delà la noésis, quel est le lien avec l'écriture, car seule la parole (ou l'absence de la parole) peut laisser une trace, un témoignage? Nous essaierons de démêler l'écheveau des problèmes en traitant, dans un premier temps, la situation narrative, avant d'aborder l'agencement spatial du roman y compris la métaphorisation de l'espace urbain en fonction de l'expérience migrante qui forme la base idéelle et du roman et de son message noétique et éthique. Nous essaierons, pour clore, de souligner certains aspects caractéristiques de l'écriture migrante d'Émile Ollivier.

\section{Configuration narrative}

La narration est subtilement disposée autour du personnage-narrateur Jonas Lazard qui, à la première personne, recolle ses propres souvenirs et commentaires en y joignant les témoignages de ses amis. La fragmentation et l'incomplétude préservent sa part de mystère à l'énigme Virgile, d'autant plus que ce personnage s'insère, in absentia, dans un curieux noyau tricéphale de la configuration des personnages où le versant subjectal, dominé par Jonas Lazard, trouve ses figures complémentaires en Dave Folentrain et Virgile, justement, celui qui, sans parole, car absent, comme Noémie, amour fatal de sa vie, est le seul dans le groupe des amis haïtiens à être désigné sans nom de famille. Si Jonas Lazard est le maître de la parole, on apprend peu sur lui, c'est une voix, un regard, une mémoire, mais dont la vie ne se laisse deviner qu'en creux des destins de Virgile et de Dave. Par contre la carrière de Dave Folentrain, poète précoce, est retracée en détail, tout comme le projet de son livre sur Montréal et la migrance. En cela, son parcours ressemble à celui de Virgile, lui aussi tenté par l'écriture et le témoignage. Or le projet de Virgile est un échec.

De trois cahiers récupérés dans la chambre de Virgile après sa disparition, je fus surpris de ne trouver que de simples commentaires sur les événements du quotidien, des considérations météorologiques ainsi que leurs répercussions sur l'âme. (Ollivier 2004 : 34)

Dave Folentrain va plus loin, prend des notes en prospectant la ville, sans pour autant mener son projet à terme :

«Avant d'aller plus loin il faut que je te dise que je viens de brûler mon manuscrit.» Je sursautai :

« Tu criais pourtant que la publication de ce livre te permettrait de t'évader du ghetto, » « Ô vanitas vanitatis! » s'exclama-t-il avec un geste théâtral, les paumes de ses mains grandes ouvertes tournées vers le ciel. [...] « J’y reprenais le thème de la flânerie. La Modernité, jusqu'à une date récente, a été le produit de la sédentarisation. De nos jours, la circulation reprend, une circulation désordonnée, tourbillonnesque même, qui ne laisse rien ni personne indemne. Elle brise les carcans et les limites établies. (Ollivier 2004 : 52)

Seul Jonas réussit le projet littéraire, le roman La Brûlerie, lui dont on sait peu de choses, sauf qu'il regarde, réfléchit et parle en inscrivant son écriture dans un dense réseau de références 
culturelles et littéraires - une quarantaine de noms (d'Homère, Virgile, Lucrèce à Zola, Pessoa, Kafka, Benjamin, Thomas Mann, Conrad, Yeats) en une trentaine d'occurrences intertextuelles qui jalonnent le texte. La configuration tricéphale du «noyau narrateur » - le vide d'une vie à retrouver (Virgile), d'une vie qui se pense incapable de la parole et aboutit au vide (Dave), et d'un vide qui se remplit de paroles jusqu'à se hisser à l'existence, la sienne et des autres (Jonas Lazard) - indique l'importance du langage : importance référentielle, bien sûr, mais surtout noétique, comme l'indique la dimension symbolique de l'anthroponymie et de la toponymie, denses de significations. Le manuscrit brûlé de Dave fait le lien avec le nom du café La Brûlerie tout comme le salut que les personnages s'échangent en créole :

« Nap boulé », répondons-nous presque en chœur. Pourquoi répondre « On brûle » quand on nous demande de nos nouvelles? Ces mots viennent probablement de loin, du fin fond de lété perpétuel, l'été noir et radieux où même l'ombre est saturée de braises. Ils viennent sans nul doute d'encore plus loin, de la condition mortelle de l'homme, de la pénitence des pécheurs et enfin du secret espoir que tel le phénix nous finirons par renaître de nos cendres. (Ollivier 2004 : 233)

Le nom de Folentrain - «Dave le fou » (Ollivier 2004 : 46) est parlant, tout autant pour la folie que pour l'allusion au roi-poète David. La dimension «poétique » de Virgile, lui aussi frappé de folie, mais d'une autre sorte, à la mort de Noémi, est corroborée par des allusions à l'Énéide (Ollivier 2004 : 56) mais aussi à l'épitaphe de sa tombe

Un jour de poisse, il avait fait promettre [Virgile] à Jacques Pellissier de lui construire une belle tombe en marbre et d'y graver une sobre épitaphe en lettres d'or rouillées. Il escomptait déjà le bel effet que cela ferait. CI-GÎT VIRGILE/ NÉ DANS LES FLAMMES DU SIÈCLE/ MORT SANS AVOIR VENDU SON ÂME (Ollivier 2004 : 91)

Jonas Lazard, celui qui « ne cesse de mourir et de renaître dans Côte-des-Neiges » (Ollivier 2004 : 9) se situe entre les deux, le mort et le vivant, en offrant par là l'image même du seul lieu possible, et qui est plutôt un non-lieu, comme on le verra plus loin, où la création puisse s'ancrer.

C’est autour de ce noyau central tripartite que se déploient les histoires et les récits des autres personnages, membres du Ministère de la Parole : docteur Barzac, Jacques Pellissier, architecte, Loana Hellebore, nom de fleur symbolique lui aussi, tout comme son nom de mariée, Volanges, qui caractérise sa liberté et sa carrière de femme fatale. Aux exilés haïtiens s'ajoute un exilé de l'intérieur, un Québécois chargé d'un nom parlant, Homère Tremblay, doublé du surnom ironique de Dionysos d'Acapulco.

La désignation - Ministère de la Parole - dégage elle aussi plusieurs significations : ironie, pour désigner ce groupe d'exilés intellectuels, déchus de la politique, dont le seul pouvoir qui reste est celui de la parole. Mais c'est aussi, en remontant l'étymologie, le ministerium, le service de la parole et, à travers la parole, le pouvoir de la parole au service de la mémoire et de l'écriture.

Plus de trente ans depuis que le Ministère de la Parole (ou ce qui en reste) se réunit dans les cafés. Nos gestes, nos paroles se comprennent dans cette répétition qui nous permet d'échapper à la 
fuite du temps. Cela fait des années que traîne sur ma table de chevet un livre d'un auteur que je vénère et qui nous exhorte à dire les mots tant qu'il y en a, à les dire jusqu'à ce qu'ils nous disent.

(Ollivier 2004 : 227-228)

C'est donc vers le noyau tripartite qui réunit un mort, autrement dit une absence et un vide que l'on cherche à remplir de mots; un poète fou, vivant, mais qui se prive de la parole en brûlant son projet; et une voix qui " ressuscite » (Ollivier 2004 : 9) par les mots des autres, vivants (amis et compagnons du Ministère), et morts (écrivains et artistes convoqués en témoignage tout au long du texte; Virgile). C'est cette métaphore de l'écriture qui s'inscrit dans le paysage montréalais, à la fois réel et symbolique, lieu de l'errance orchestrée autour du curieux avatar du guide de Dante qu'est, après tout, là comme ici, Virgile.

\section{Agencement spatial}

Le Montréal de La Brûlerie est disposé, de manière analogue à la configuration des personnages, en deux ensembles complémentaires. Le noyau, une sorte d'hétérotopie foucaultienne qui condense l'univers (Foucault 1984), est le quartier de la Côte-des-Neiges avec, au centre La Brûlerie. Ce noyau est complété par trois traversées significatives de la ville. La première est celle de Jonas Lazard qui donne une image «migratoire » de la ville en accentuant le caractère fluide, limitrophe, indécis, de centre-périphérie, faille, frontière:

J'aime me promener en autobus. En traversant Montréal en autobus, je me sens comme un enfant dans un parc d'attraction, émerveillé par tant de manèges. J'absorbe avec avidité toute la beauté de la ville et même, dans certains coins, la beauté de sa laideur. J'ai mes rues et mes circuits de prédilection. Le 24 parcourt la rue Sherbrook d'est en ouest. Sur ce trajet, on peut expérimenter physiquement les deux solitudes : l'Ouest à majorité anglophone et l'Est, bastion des francophones. Le 80 de l'avenue du Parc : un autobus brinquebalant transporte des couples d'amoureux pour les déverser sur le Mont-Royal. Le 55 longe le boulevard Saint-Laurent, rue frontière au statut particulier, rude épreuve de l'autre, au bornage indécis, contiguité du proche et du lointain. À la fois centre et périphérie, le boulevard Saint-Laurent est une faille, une erreur d'imagination, un défi lancé, avec son atmosphère de bazar, d'échange hétéroclite de biens et de signes; le remonter du sud au nord prend à chaque station l'aspect d'un voyage en terre inconnue. Je ne suis pas seulement sensible à l'évidente splendeur de la ville. Je regarde les visages, je scrute leur ennui, je comprends leur lassitude. Ah! saisir au fond d'un regard l'éclair de désespérance! Prendre la mesure d'immenses déserts privés d’oasis! (Ollivier 2004 : 61 ; souligné par nous)

La deuxième, qui suit de près la première, est l'errance nocturne de Virgile, épié par le narrateur, dans le labyrinthe forestier du Mont-Royal (Ollivier 2004 : 64-66). La troisième, raconté par Dionysos d'Acapulco à la manière d'une parabole biblique, retrace les pas de Jésus Christ revenu sur terre et qui marche dans la rue Sainte-Catherine d'ouest en est en produisant des miracles - vue rendue à un aveugle, jambes rendues à un infirme en chaise roulante : 
Envahi par un vide triste, ses pas le conduisirent machinalement jusqu'à l'angle du boulevard Saint-Laurent. Là, un homme, un Noir, accroupi, la tête entre les mains, semblait écrasé sous le fardeau de tous les malheurs du monde. Il pleurait à chaudes larmes. " Pourquoi pleures-tu ?» s’inquiéta Jésus. «Aïe, mon fils! fit l'homme, il n’y a pas de mots pour décrire ma condition. » Et il continua à pleurer. « Mais d’où viens-tu ? s'enquit Jésus. « Je suis haïtien », dit l'homme entre deux sanglots. Jésus hocha la tête de haut en bas en signe de compréhension. Puis, relevant sa tunique, il s'assit à côté de l'homme; ils pleurèrent de concert toute la nuit, jusqu'à l'aube. [...] Nouvelles lamentations de la Passion. Le Père avait une fois livré au désespoir son fils qui se faisait, en mêlant ses larmes à celle de l'Haïtien, l'aveu de son irréalisable entreprise de sauver l'humanité. Mon père, pourquoi m’avez-vous abandonné ? (Ollivier 200 : 174-175)

L'espace, on le voit, se charge de significations symboliques. En contrepoint des traversées se situe le lieu de concentration - le quartier de la Côte-des-Neiges. Sur le plan réel, géographique, il s'agit d'une sorte d'au-delà, car situé de l'autre côté du Mont-Royal par rapport au centre qu'est le Vieux-Montréal et les rues centrales parcourues par les traversées. C’est cette périphérie qui se constitue en centre, avec son Université, son lieu de pèlerinage - Oratoire Saint-Joseph, son hôpital, son marché, son musée de cire. Les cafés et les restaurants que les membres du Ministère de la Parole élisent pour siège (Ollivier 2004 : 19-27) à tour de rôle avant de se fixer à La Brûlerie sont à l'image des vagues migratoires qui inondent le quartier.

La toponymie, encore, est parlante. Si la saveur du café torréfié à La Brûlerie évoque les Caraibes, la neige, évoquée par le toponyme du quartier, rappelle le nord, le lieu de l'exil, alors que la côte, du même toponyme, peut se rapporter aussi bien à la montagne montréalaise qu'au rivage haïtien. Montréal est une île, au fond.

Ainsi, ce lieu de concentration est aussi un lieu transitoire. La métaphorisation de la topique est hautement évocatrice :

Je ressuscite depuis des décennies dans Côte-des-Neiges [... ]. Pendant que je ne cesse de mourir et de renaître dans Côte-des-Neiges, que n'ai-je point vu? Maints vols d'outardes et leurs vagues ondulées quand elles reviennent de leurs campements d'été. Les brouillards et les brumes enveloppant le mont Royal tandis que la neige avance à pas feutrés. Chemin de la Côte-de-Neiges, j'ai vu passer une foule de papillons multicolores, le monde réel : plaisirs, bonheurs, espérances et chaque pouce d'asphalte, un parterre de fleurs. J'ai vu des quantités de Bédouins caracolant sur leur chamelle de transhumance, narines au vent. D’où viennent ces pèlerins fluides et froids qui s'arrêtent aux terrasses des cafés pour discuter, se disputer, douter et continuer leur chemin, traversés et portés par tous les souffles de la Terre, de l'Eau, du Feu et du Vide ? J'ai vu ces peuples des espaces intermédiaires. Chassés de leur communauté, ces cohortes de flottants ont choisi de vagabonder, poussés par le vent : guerriers en rupture de guerre, saltimbanques sans audience, professionnelles de la retape, moines errants accompagnés de nonnes mendiantes qui offrent des images pieuses figurant l'enfer et le paradis en tendant leur sébile pour l'aumône. Les trottoirs de la Côte-des-Neiges ont résonné de leurs voix [...] des milliers de voix qui semblent sourdre des entrailles de la terre. (Ollivier $2004: 9-10)$ 
L'endroit, subtilement placé sous le signe de la migration des papillons et des oiseaux, relie la nature à la transhumance et à la migration humaine, le paysage urbain à la nature et, par-delà la nature, aux quatre éléments de l'imaginaire bachelardien, universel, mais où l'air est pour le moment remplacé par le Vide, alors qu'il revient sous forme du vent métaphorique, avec l'eau suggérée par les « cohortes de flottants », image récurrente tout au long du roman, où la vision complexe d'Haïti/mer se mêle à celle de Montréal/neige et où l'image de l'eau, de la vague, de la fuite, se joint à celle du feu, de la brûlure, du malaise existentiel :

À la Côte-des-Neiges, il ne manque que le port, la rouille des cargos, les grues géantes, le ronflement des chalands, les cales sèches et, au loin, l'horizon bleuté. Vous vous demandez pourquoi j'aime les terrasses des cafés. Probablement parce que je suis nostalgique des baignades, des mares à crevettes, des eaux impures, des bords de plages où flottent des carcasses de crabes. [...] Je crois l'avoir déjà dit, nous sommes des naufragés - tous autant que nous sommes. [...] Les vagues d'émigrants se succèdent et ne se ressemblent pas. Nous sommes tous des naufragés. Notre mémoire n'est pas seulement un paradis perdu, elle porte la marque de la brûlure d'un enfer d'où nous nous sommes échappés. Et depuis, projetés dans un monde de chaos, nous sommes voués à l'errance, formant ainsi un troupeau de Bédouins qui ne connaissent pas d'autres lois que le respect, l'échange et la complicité des sentiments. (Ollivier $2004: 69-71$ )

\section{Vide}

Avec une dizaine d'occurrence, le thème du vide s'attache tantôt aux personnes :

Naomi est nue devant la fenêtre qui donne sur un environnement urbain [...]. Ses yeux semblent regarder au-delà de la fenêtre, au-delà du vide. [...] elle est comme exilée dans le cône de lumière qui projette un carré clair sur le plancher de la chambre. (Ollivier 2004 : 203)

tantôt au paysage urbain de la ville - tel cet « escalier suspendu au-dessus du vide, pans de murs mal démolis, fragments de briques » (Ollivier 2004 : 246). Dans les deux cas le vide est un sentiment étroitement lié à l'exil :

[...] nous avions convenu que cette ville faisait planer sur nous une menace, et la première, la principale, en un lieu tel que Montréal, c'était le vide : une crainte permanente, l'obsession d'être aspirés par cette sorte d'ozone spirituel, cette eau de Javel qui nous blanchissait subrepticement. (Ollivier $2004: 41$ )

Et cela au point de devenir un absolu d'allure métaphysique :

Au commencement était le Vide. Et il ne s’est rien passé par la suite. Ni création ni surgissement, aucun événement. Rien à signaler. (Ollivier 2004 : 25)

Absence de Dieu? Déréliction? Pas tout à fait. La Brûlerie n'est pas un roman doloriste, mais une réaction à un mal d'être, au malaise existentiel de l'exil : 
« L'exil est une perte de consistance. Au point de départ on fait l'expérience de la vacuité, de la légèreté, de l'absence de pesanteur. On croit déboucher sur la liberté, mais cette liberté est accompagnée d'indifférence et progressivement de détachement. Il faut prendre acte de cette disjonction, il faut prendre acte du malheur qu'est l'exil, de cette tristesse, du vide qui nous prive de notre être, de cette impuissance à agir », n’arrêtait-il de répéter. Cette forme de détachement que Dave a été, degré par degré, amené à pratiquer, il la nommait « déliaison » [...]. « La déliaison, c’est ce que devient l’être dépossédé de toute attache. [...] Tout compte fait, la déliaison, c’est flotter au hasard. Comment sortir de cette vacuité dissolvante ? Comment retrouver le courage de l'action, lutter contre la funeste disparition? »(Ollivier 2004 : 166-167)

Le sentiment du vide existentiel, on le voit, semble davantage exprimer l'absence de l'essentialité. Le vide caractérise une existence sans attaches, déliée, située dans un entre-deux, à la limite, aux frontières, une existence dynamisée par le peu de consistance, voire par l'inconsistance des limites mêmes qui changent et se déplacent, flottantes, fluides. La ville même de Montréal s'y prête :

Montréal, tu parais une œuvre de hasard plutôt qu'une créature de l'homme. Qui es-tu, ville élue? Hermaphrodite, tu es un hermaphrodite te promenant nu au bord d'un fleuve ! [...] «Connaissez-vous un autre endroit où, parvenu à l'extrême limite du continent, on peut en contempler un autre, par delà un bras d'eau ?»(Ollivier 2004: 42)

Le thème de la limite revient presque aussi souvent, dans le texte, que celui du vide, et les deux s'associent à la thématique de l'exil envisagé soit comme un vécu négatif - errement ou errance, soit neutre, voire positif - migrance.

\section{4. Écriture migratoire?}

Comment sortir du piège de l'exil ? Le témoignage par l'écriture est non seulement une thérapie, mais également une noésis de la situation existentielle. Tel est du moins le projet de Dave Folentrain qui veut " mettre des mots sur le désordre, sur le chaos d'un monde » (Ollivier 2004 : 53).

Or la nature des mots est glissante, ambiguë, comme l'indique la dispute entre Virgile et Naomie :

Alors qu'au cours du déjeuner il soutenait que les mots sont des faits, qu'ils servent, à partir de la périphérie, à trouver, le centre des choses, elle lui avait répliqué qu'elle ne croyait pas aux mots, qu'ils ne font que meubler le vide de la déréliction, que seul compte le silence. (Ollivier 2004 : 193)

L'échec existentiel de Virgile et de Noémie, écrasés par la réalité de l'Histoire, et l'échec de Dave Folentrain qui, fasciné, comme Virgile et Noémie, par l'essentialité de la parole (ou du silence), abdique devant la réalité, sont assumés par la voix de Jonas Lazard : 
Je lui dis aussi [à Dave Folentrain] qu'un vrai travail d'écriture sur Montréal devrait commencer par mettre en scène la parole nomade, la parole migrante, celle de l'entre-deux, celle de nulle part, celle d'ailleurs ou d'à côté, celle de pas tout à fait d'ici, pas tout à fait d'ailleurs; [...]. (Ollivier 2004 : 55-56)

Ainsi, une autre écriture s'instaure, celle de l'entre-deux, entre la présence et l'absence, entre la parole et le silence, entre le lieu et le non-lieu. Une absence qui se fait présence : le Ministère de la Parole, au sens de service, et qui consiste à « dire les mots tant qu'il y en a, à les dire jusqu'à ce qu'ils nous disent » (Ollivier $2004: 228$ ).

Ainsi, une sorte d'analogie structurelle se dessine entre la configuration narrative (VirgileDave-Jonas), la narration et l'inscription de la parole dans l'espace/temps. Si jusqu'ici la temporalité n'a pas fait l'objet de notre analyse, ce n'est pas faute de sa thématisation dans le roman. $L a$ Brûlerie l'attache à la mémoire :

Ce qui se réalise dans l'histoire de l'île [Haïti] n'est pas le passé simple de ce qui fut et qui n'est plus, ni même le parfait de ce qui a été, mais le conditionnel de ce que nous aurions pu être devant ce que nous sommes. [...] Cette histoire est la figure de l'oubli, notre dernière demeure; son écriture est motivée par le désir de fixer des limites, d'épingler les signes du temps sur les traces de l'oubli. Les jeux de miroirs, de redoublement ou de diffraction dessinent les frontières qui séparent l’oubli de la vérité, qui séparent Léthé d'Alétheia. Déjà les anciens avaient pressenti que Léthé est compagnon d'Éros. (Ollivier 2004 : 36-37)

Or, on le voit, c'est encore une histoire conditionnelle, soit irréelle dans le cas d'Haïti, soit potentielle si elle est tournée vers le présent ou l'avenir et qui se joue entre la mémoire/vérité et l'oubli/silence. Il est encore question de frontières et de limite. La parole joue encore le rôle central comme instrument. Dans La Brûlerie, elle travaille l'espace d'une manière toute particulière : au lieu de le segmenter en topoï sémantiquement distincts elle tend à le dynamiser en le présentant soit sous forme de lieux transitoires de concentration (les cafés dont La Brûlerie), soit comme des espaces dynamiques complémentaires du lieu de concentration (traversées de Montréal). S’y ajoute la métaphorisation de l'espace qui joue, encore, dans le sens de l'intégration sémantique dynamisante des valeurs. Cette variante de l'hétérotopie foucaultienne va justement dans le sens d'une narration potentielle construite, par la parole, autour du vide d'une vie (Virgile), autour d'un pays absent (Haït), dans un pays inconsistant et qui n'en est pas un (Québec), autour de destinées flottantes, sans lieux fixes, nomades. La Brûlerie, le café et le roman, à la fois réalité et métaphore de la création, constituent

un espace-temps clos qui, dans son déroulement, abolit la linéarité du temps, leur permet [aux membres du Ministère de la Parole] de ne pas le contrer directement, de le tromper, d'échapper à l'inévitable érosion et ainsi arpenter un univers stable, ordonné, sans miracle, sans métamorphose. Ils habitent la terrasse de La Brûlerie, après avoir promené leur gueule dans tous les cafés du coin. Ils ont découvert leur café, comme Verlaine, le Procope, Pessoa, l'Arcada, Kafka, le Savoy. (Ollivier $2004: 236$ )

Le rattachement de la vie narrée à la vie littéraire est significatif du jeu de la limite, de l'entredeux. 


\section{Conclusion}

Le dernier roman d'Émile Ollivier met en œuvre certaines des idées qu'il a formulées dans Repérages (2001), dont l'essai central s'intitule « Ontologie d'un exil ». Il y constate le changement du paradigme : si à un certain moment de l'histoire de l'humanité le nomadisme a été supplanté par la sédentarité comme facteur culturel dominant, la postmodernité renverse la tendance, relativise la sédentarité et accentue de nouveau le nomadisme qu’il s'agisse de migrations économiques, d'exils politiques ou de transferts culturels caractéristiques de la mondialisation (Ollivier 2001 : 31 sqq.). En se référant à Věra Linhartová et son article «Pour une ontologie de l'exil »(Ollivier 2001 : 33 sqq.), il souligne les conséquences positives de la migration, à la fois sur le plan personnel (liberté) et créateur (enrichissement noétique et culturel). Sa conception de la littérature migrante prend pour point de départ la littérature mineure de Gilles Deleuze (Ollivier 2001 : 52 sq.), mais ce rappel obligé n’est que le préliminaire d'une réflexion fondamentale dont le noyau se compose de plusieurs concepts : celui de l'identité en fusion, une identité dynamique, changeante, anti-essentialiste (opposée à celle de Fernand Dumont, Ollivier 2001 : 58), ceux de l'invention de soi (Ollivier 2001 : 74 sqq.) et de la mémoire (Ollivier 2001 : 92 sqq.), celui de l'écriture en périphérie (Ollivier 2001 : 96 sqq.) et surtout de l'espace littéraire médian (Ollivier 2001 : 104 sqq.).

Certaines de ces notions, nous avons pu le constater, s'inscrivent dans la stratégie narrative originale du romancier et qui reflète une esthétique de l'éphémère, du changeant. La différence n'est pas dans le changement radical des catégories narratives que nous avons traitées, mais dans l'axiologie, autrement dit dans les valeurs attribuées à ces catégories et dans leur interprétation.

\section{Références bibliographiques}

\section{Ouvrages d'Émile Ollivier}

Ollivier, É. (1987). Être d'ici. Nuit blanche, le magazine du livre, 28, 46-47.

. (2001). Repérages. Ottawa : Leméac.

- (2001). Autoportrait en cheval fou. Lettres québécoises : la revue de lactualité littéraire, 102, 7.

(2004). La Brûlerie. Montréal : Boréal.

. (2002). L'enracinement et le déplacement à l'épreuve de l'avenir. Études littéraires, 34, 3, 87-97.

\section{Ouvrages consultés}

Février, G. (2009). Entrevue avec Émile Ollivier. Québec français, 152, 74-75.

Foucault, M. (1984). Des espaces autres. Architecture, Mouvement, Continuité, 6 (octobre), 46-49.

Deleuze, G. (1975). Kafka, pour une littérature mineure. Paris : Minuit.

Jonassaint, J. (1992). Émile Ollivier : Écrire pour soi en pensant aux autres. Lettres québécoises : la revue de l'actualité littéraire, 65, 13-15.

Linhartová, V. (1994). Pour une ontologie de l'exil. Atelier du roman, 2 (mai). 
Lotman, J. M. (1990). Štruktúra umeleckého textu. Bratislava : Tatran. (Édition russe : Struktura chudožestvennogo teksta, Moskva : Iskusstvo, 1970.)

Pauyo, É. (2002). L’exil et le royaume : entretien avec Émile Ollivier. Spirale : arts, lettres, sciences humaines, 182, 8-9.

Péan, S. (2001). Émile Ollivier : entre nostalgie et lucidité. Lettres québécoises : la revue de lactualité littéraire, 102, 11-12.

. (2003). Émile Ollivier (1940-2002) : peindre le passage. Lettres québécoises : la revue de l'actualité littéraire, 109, 7.

Selao, C. (2005). Roman de l'exil et exil du roman. Ouvrages recensés : La Brûlerie d'Émile Ollivier, Boréal, 246 p. ; La ligne gothique de Fulvio Caccia, Triptyque, 153 p. Spirale : arts, lettres, sciences humaines, 201, 16-17.

Spear, T. C. (2002). Émile Ollivier : enracinerrant de Notre-Dame-de-Grâce. Études littéraires, 34, 3, $15-$ 27.

Voldřichová Beránková, E. (2004). La topographie de la mort chez Patrick Modiano. In Rencontres françaises - Brno 2003. Brno : Masarykova univerzita. 
